\title{
Correlation of EGFR Del 19 with Fn14/JAK/STAT signaling molecules in non-small cell lung cancer
}

\author{
YING SUN*, YONG HAN*, XIAOPING WANG*, WUPING WANG, XUEJIAO WANG, \\ MIAOMIAO WEN, JINGHUA XIA, HAO XING, XIAOFEI LI and ZHIPEI ZHANG
}

Department of Thoracic Surgery, Tangdu Hospital, The Fourth Military Medical University, Xi'an, Shaanxi 710038, P.R. China

Received January 30, 2016; Accepted May 30, 2016

DOI: $10.3892 /$ or.2016.4905

\begin{abstract}
Previous research has shown that p-EGFR (particularly mutated EGFR) may activate fibroblast growth factor-inducible 14 (Fn14) expression in non-small cell lung cancer (NSCLC), and the JAK/STAT signaling pathway may participate in this process. Thus, in order to verify this hypothesis, correlations among the expression levels of EGFR Del 19, Fn14 and JAK/STAT were detected and analyzed. The expression and location of these molecules were assessed using IHC, immunohistofluorescence, RT-qPCR and western blotting. The differences and correlations in the expression of these molecules and clinical pathological characteristics were statistically analyzed using Mann-Whitney U, Kruskal-Wallis $\mathrm{H}$ and cross-table tests. Kaplan-Meier survival analysis and Cox proportional hazards models were used to estimate the effect of EGFR Del 19 and Fn14 expression on survival. Data showed that EGFR Del 19, Fn14 and JAK1/STAT1 expression was significantly related with differentiation, pTNM stage and lymphatic metastasis $(\mathrm{P}<0.01)$ and there was a marked correlation of EGFR Del 19, Fn14 and JAK1/STAT1 expression with histological type, differentiation, pTNM stage of NSCLC $\left(\mathrm{P}<0.05 ; \mathrm{r}_{\mathrm{s}}>0.3\right)$. Immunohistofluorescence showed that there was a co-localization phenomenon between EGFR Del 19 and Fn14 expression. NSCLC patients with higher EGFR Del 19/Fn14 expression had a significantly worse prognosis than those with lower EGFR Del 19/Fn14 expression $(\mathrm{P}=0.0155 / \mathrm{P}=0.001 ; \log$-rank test $)$. The multivariate analysis indicated that Fn14 expression may be an independent prognostic factor in NSCLC with EGFR Del 19 [hazard ratio
\end{abstract}

Correspondence to: Professor Xiaofei Li or Professor Zhipei Zhang, Department of Thoracic Surgery, Tangdu Hospital, The Fourth Military Medical University, 1 Xinsi Road, Xi'an, Shaanxi 710038, P.R. China

E-mail: 837730550@qq.com

E-mail: zzpzyy@fmmu.edu.cn

${ }^{*}$ Contributed equally

Key words: non-small cell lung cancer, EGFR exon 19 deletion, Fn14, JAK1, STAT1
(HR), 0.326; $\mathrm{P}=0.042]$. Therefore, our results indicate that EGFR Del 19 may promote Fn14 and JAK1/STAT1 expression in NSCLC and Fn14 may serve as a prognostic biomarker in NSCLC with EGFR Del 19.

\section{Introduction}

Lung cancer is the leading cause of cancer-related deaths in China for both men and women, and $\sim 85 \%$ of these cases are non-small cell lung cancer (NSCLC) (1). Research indicates that disorders of signaling pathways such as epidermal growth factor receptor (EGFR) may lead to NSCLC progression. EGFR is overexpressed or mutated in $60-70 \%$ of NSCLC, but less in normal lung tissues (2-4). The most common EGFR mutation is exon 19 deletion (EGFR Del 19), accounting for $45 \%$ of EGFR mutations (5), and EGFR Del 19 may upregulate the downstream signaling pathway and promote new signaling molecular expression, leading to tumor proliferation and metastasis. Although NSCLC patients with EGFR Del 19 often markedly respond to EGFR tyrosine kinase inhibitors (TKIs) (6), high cost and emergence of acquired resistance are virtually inevitable, thus limiting the therapeutic effects in these patients. In order to solve this issue and identify a new therapeutic target for the treatment of NSCLC with EGFR Del 19, it is necessary to research downstream molecular mechanisms when EGFR is mutated.

Fibroblast growth factor-inducible 14 (Fn14; gene TNFRSF12A) is the smallest member of the tumor necrosis factor (TNF) receptor family and is induced in tissue-injured situations (7,8-12). Low expression of Fn14 has been reported in fibroblasts, endothelial and epithelial cells of healthy homeostatic tissue, but is overexpressed in damaged tissues, such as tumors (13-15). Whitsett et al (16) showed that Fn14 is frequently overexpressed in NSCLC cell lines, and it is correlated with p-EGFR expression. In addition, it may promote tumor proliferation, invasion and migration through the JAK/ STAT pathway (17). Given all these elements, we speculated that EGFR Del 19 may promote NSCLC cell survival and proliferation through the Fn14/JAK/STAT pathway, but the activation mechanism is unclear. Thus, the present study focused on the correlation of EGFR Del 19 and Fn14 signaling pathway expression in NSCLC in order to provide a basis for further study on the developmental mechanism of EGFR Del 19 in regards to NSCLC. 


\section{Materials and methods}

Patients and tissue samples. Paraffin-embedded tissue specimens from 343 patients with confirmed NSCLC (including 125 EGFR Del 19, 100 L858R mutations as well as 118 wild-type EGFR selected by ARMS, and the latter two as comparisons) and 30 corresponding normal lung tissues, collected from 2010 to 2013, were analyzed from an archived thoracic oncology tissue repository at the Department of Thoracic Surgery of Tangdu Hospital affiliated with The Fourth Military Medical University. Patients who received preoperative chemotherapy, radiotherapy or EGFR-targeted therapy were excluded from the present study. Detailed information was collected from the medical records of the enrolled patients in a computerized registry database including patient age, gender, smoking history, clinical manifestation, surgical method, tumor status, histological differentiation, nodal status and follow-up information. The follow-up lasted until September 30, 2014, with a median follow-up period of 21.65 months for living patients (range, 7.43-48.17 months). The day of surgery was considered as the starting day for estimating postoperative survival time. Histological classification of tumors was reviewed by pathologists and based on the World Health Organization criteria. All tumors were staged according to the pathological tumor/node/metastasis (pTNM) classification (7th edition) of The World Health Organization. The study protocol was approved by the Regional Ethics Committee for Clinical Research of the Fourth Military Medical University. All patients provided written informed consent for use of their medical records and tissue specimens for research purposes.

Immunohistochemistry. The tumor samples were fixed with $10 \%$ formaldehyde and embedded with paraffin. Sections were sliced to $4-\mu \mathrm{m}$ thickness, deparaffinized with a series of xylene and rehydrated through a graded series of alcohol. Microwave antigen retrieval was performed at $750 \mathrm{~W}$ for $5 \mathrm{~min}$ and $450 \mathrm{~W}$ for $15 \mathrm{~min}$ in citrate buffer $(\mathrm{pH}$ 6.0) to enhance the immunoreactivity. After blocking the endogenous peroxidase activity with $3 \%$ hydrogen peroxidase for $30 \mathrm{~min}$, the sections were incubated with 5\% normal goat serum for $30 \mathrm{~min}$ at room temperature to block non-specific antibody reaction. After washing the tissue samples with phosphate-buffered saline (PBS) three times for $5 \mathrm{~min}$, the sections were incubated with the primary antibodies [EGFR Del 19 at 1:100 (Cell Signaling Technology, Beverly, CA, USA); Fn14 at 1:100 (Abcam, Cambridge, UK); p-JAK1 at 1:100; p-STAT1 at 1:100 (both from Abcam)] overnight at $4^{\circ} \mathrm{C}$, and incubated with an EnVision ${ }^{\mathrm{TM}}$ Detection kit (Dako, Glostrup, Denmark) following the manufacturer's instructions. The sections were then reacted with $0.003 \%$ 3,3'-diaminobenzidine and counterstained with hematoxylin. To confirm the specificity of the immunostaining, negative controls were obtained by replacing the primary antibody with PBS.

Evaluation of the immunohistochemical (IHC) staining. Five random fields from each section were viewed under a light microscope (Leica DM4000B; Leica, Wetzlar, Germany) in a high-power field (magnification, $\mathrm{x} 400$ ). We scored all sections in accordance with previous studies on IHC staining $(18,19)$.
Briefly, a total immunostaining score was calculated as the product of a proportion score and an intensity score. The proportion score represented the estimated fraction of positively stained tumor cells $(0,0-5 ; 1,6-25 ; 2,26-50 ; 3,51-75$ and $4,76-100 \%$ ). The intensity score represented the estimated staining intensity $(0$, negative; 1 , weak; 2 , moderate; 3 , strong). The score of these were measured according to the result of the degree multiplied by the score of the staining intensity: 0,0 ; $1+, 1-4 ; 2+, 5-8$ and $3+, 9-12$. Score 0 was considered negative, whereas scores (1+ to $3+)$ were considered positive. Thus, the total score ranged from 0 to 12 . All slides were assessed by two independent investigators who were blinded to the clinical features and outcomes. The final IHC staining score reported is the average of the scores from the two investigators.

$R T-q P C R$. RT-qPCR was performed for gene expression detection in specimens of NSCLC with EGFR Del 19 and corresponding normal lung tissues. Total RNA was isolated using the E.Z.N.A. Total RNA Kit I Bio-Tek (R6834; Omega Bio-Tek, Norcross, GA, USA) and reverse-transcribed to cDNA using RevertAid First Strand cDNA Synthesis kit (K1622; Thermo Fisher Scientific, Vilnius, Lithuania). The primers were as follows: forward primer, 5'-CCA AGC TCC TCC AAC CAC AA-3' and reverse primer, 5'-TGG GGC CTA GTG TCA AGT CT-3' for Fn14 gene; forward primer, 5'-GGG AAA TCT GCT ACA ATG GC-3' and reverse primer, 5'-TGA TGG CTC GGA AGA AAG GC-3' for JAK1 gene; forward primer, 5'-GTT GAA CCC TAC ACG AA-3' and reverse primer, 5'-TAC AGA GCC CAC TAT CC-3' for STAT1 gene; forward primer, 5'-GAG CTA CGA GCT GCC TGA CG-3' and reverse primer, 5'-CCT AGA AGC ATT TGC GGT GG-3' for $\beta$-actin gene. $\beta$-actin served as the internal control.

Western blotting. Cytoplasmic and nuclear protein were extracted from the tissues using RIPA lysate (P0013B; Beyotime Institute of Biotechnology, Jiangsu, China) and western blotting was performed using the EGFR Del 19 monoclonal antibody (6B6; Cell Signaling Technology), Fn14-specific monoclonal antibody (EPR3179), p-JAK1 monoclonal antibody (EPR1899), p-STAT1 monoclonal antibody (EPR3146) (all from Abcam) and the $\beta$-actin-specific polyclonal antibody (CW0097; CW Biotech Co., Ltd., Beijing, China).

Protein concentrations were determined using the BCA assay kit (Pierce Biotechnology, Inc., Rockford, IL, USA). Equivalent amounts of each protein sample were mixed with loading buffer (CW0027A; CWBio, Beijing, China), heated at $65^{\circ} \mathrm{C}$ for $30 \mathrm{~min}$, and subjected to SDS-PAGE using 12\% separation and 5\% spacer gels. Then the protein was transferred to a polyvinylidene fluoride (PVDF) membrane (Solarbio, Beijing, China) by electroblotting (Bio-Rad, Philadelphia, PA, USA). The membrane was blocked for $3 \mathrm{~h}$ at room temperature in Tris-buffered saline and Tween-20 (TBST) $(25 \mathrm{mM}$ Tris/HCl, $\mathrm{pH} 7.5,150 \mathrm{mM} \mathrm{NaCl}, 0.1 \%$ Tween-20) containing $5 \%$ non-fat dry milk. The membranes were incubated overnight at $4^{\circ} \mathrm{C}$ in 1:2,000 dilution of anti-EGFR Del 19 monoclonal antibody, 1:5,000 dilution of anti-Fn14 monoclonal antibody, 1:5,000 dilution of p-JAK1 monoclonal antibody, 1:7,500 dilution of p-STAT1 monoclonal antibody and 1:2,500 dilution of $\beta$-actin polyclonal antibody with WB Antibody Diluent (P0023A; Beyotime Institute of Biotechnology), washed six times in 
TBST, and incubated for $35 \mathrm{~min}$ in a 1:5,000 dilution of goat anti-rabbit Ig-HRP (EK020; Zhuangzhi Bio, Xi'an, China) with WB Secondary Antibody Diluent (P0023D; Beyotime Institute of Biotechnology). Immunoreactive bands were revealed by the enhanced chemiluminescence system (Santa Cruz Biotechnology, Santa Cruz, CA, USA). Images were captured and analyzed by GelDox XR system (Bio-Rad).

Immunohistofluorescence. The tumor samples were fixed with $10 \%$ formaldehyde and embedded with paraffin. Sections were sliced to $4-\mu \mathrm{m}$ thickness, deparaffinized with a series of xylene and rehydrated through a graded series of alcohol. Microwave antigen retrieval was performed at $750 \mathrm{~W}$ for $5 \mathrm{~min}$ and $450 \mathrm{~W}$ for $15 \mathrm{~min}$ in citrate buffer $(\mathrm{pH}$ 6.0) to enhance the immunoreactivity. After blocking the endogenous peroxidase activity with $3 \%$ hydrogen peroxidase for $30 \mathrm{~min}$, the sections were incubated with $5 \%$ normal goat serum for $30 \mathrm{~min}$ at room temperature to block non-specific antibody reaction. After washing the tissue samples with PBS three times for $5 \mathrm{~min}$, the sections were incubated with the primary antibodies [EGFR Del 19 at 1:100 (Cell Signaling Technology); Fn14 at 1:100; p-JAK1 at 1:100; p-STAT1 at 1:100 (all from Abcam)] overnight at $4^{\circ} \mathrm{C}$. After an additional series of washes, the slides were stained with goat anti-rabbit (Cy3; Zhuangzhi Bio, Xi'an, China) at room temperature for $50 \mathrm{~min}$, and then the tissue samples were washed with PBS three times for $5 \mathrm{~min}$. The sections were then incubated with the other primary antibodies overnight at $4^{\circ} \mathrm{C}$. After an additional series of washes, the slides were stained with goat anti-mouse (Alexa Fluor 488; Zhuangzhi Bio) at room temperature for $50 \mathrm{~min}$. After the final washing, the slides were mounted in 50\% glycerol (in PBS) and examined by a fluorescence microscope (Leica DM4000B).

Statistical analysis. As our data were not normally distributed, we used non-parametric tests. The degree of protein expression in the primary tumor was compared by cross-table analysis. The expression differences among more than three groups were analyzed by Kruskal-Wallis H test, otherwise MannWhitney U analysis was used. Survival curve was examined using the Kaplan-Meier method, and correlation analyses of the survival time and various clinicopathological variables were performed by univariate and multivariate analyses using the Cox regression model. All analyses were performed using SPSS 15.0 software (SPSS, Inc., Chicago, IL, USA). P-values were adjusted for multiple testing and a $\mathrm{P}<0.05$ was considered to indicate a statistically significant result. Spearman's rank correlation coefficients were calculated for the assessment of overall concordance, and $r_{s}$ was used to express the relevant coefficient, and when $\mathrm{P}<0.05, \mathrm{r}_{\mathrm{s}}>0.3$ was considered positive relevance.

\section{Results}

Patient characteristics. The clinicopathological characteristics of the NSCLC patients with EGFR Del 19 are summarized in Table I. There were 62 female and 63 male patients with a median age of 58 years (range, 36-79 years). The patients were diagnosed with squamous cell carcinoma (SCC) $(n=30,24 \%)$ and adenocarcinoma (ADC) $(\mathrm{n}=95,76 \%)$. Histopathologic diagnosis included: well differentiated $(n=23,18.4 \%)$, moder-
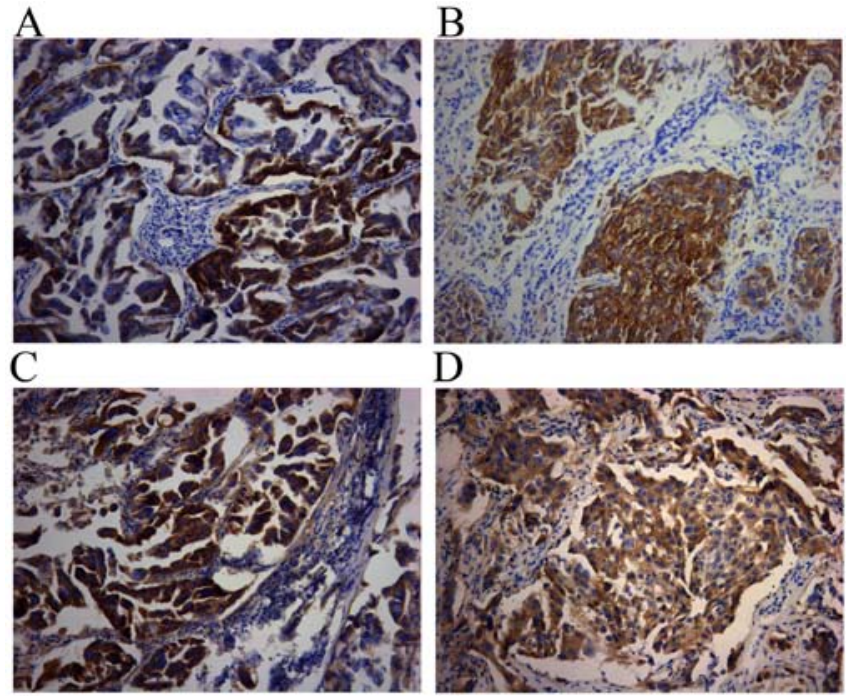

E

$\mathrm{F}$
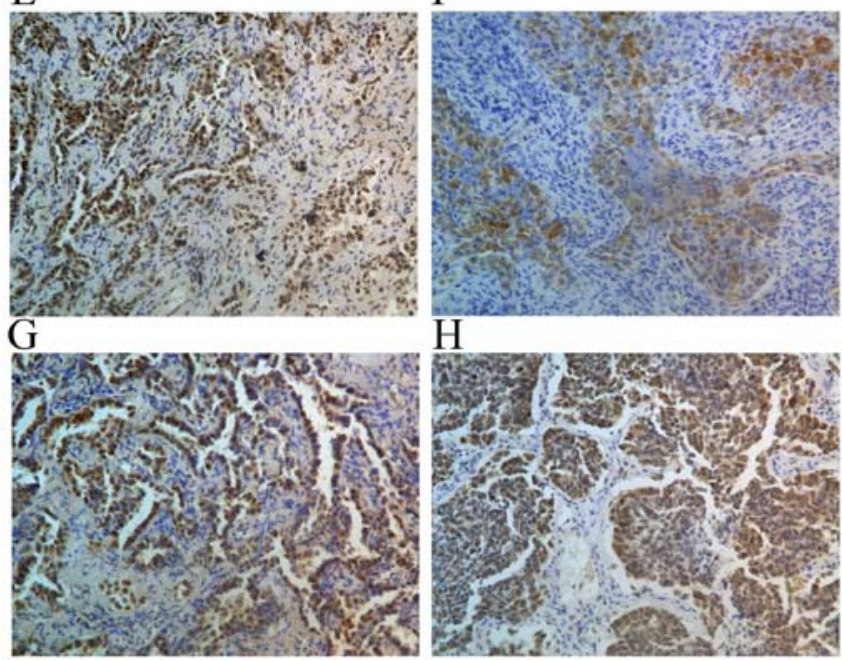

$\mathrm{H}$

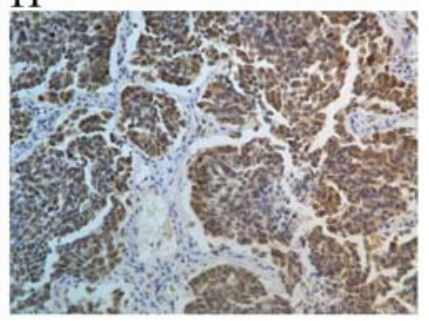

Figure 1. Expression of EGFR Del 19, Fn14, p-JAK1 and p-STAT1 by immunohistochemistry in NSCLC samples. (A) Positive EGFR Del 19 staining in ADC. (B) Positive EGFR Del 19 staining in SCC. (C) Positive Fn14 staining in ADC. (D) Positive Fn14 staining in SCC. (E) Positive p-JAK1 staining in ADC. (F) Positive p-JAK1 staining in SCC. (G) Positive p-STAT1 staining in ADC. (H) Positive p-STAT1 staining in SCC (original magnification, x200). ADC, adenocarcinoma; SCC, squamous cell carcinoma.

ately differentiated $(\mathrm{n}=83,66.4 \%)$ and poorly differentiated $(n=19,15.2 \%)$ tumors. Postoperative staging evaluation demonstrated stage I disease in 43 patients, stage II disease in 19 patients, stage III disease in 57 patients and stage IV disease in 6 patients.

Protein expression in NSCLC with EGFR Del 19 and the correlation with clinicopathological parameters. In the present study, 99.2\% (124/125) of the tumor sections were classified as EGFR Del 19-positive as detected by IHC and positive staining was mainly located in the cytoplasm (Fig. 1A and B). The correlation of EGFR Del 19 expression with clinicopathological parameters was then investigated. EGFR Del 19 expression was significantly associated with differentiation $(\mathrm{P}<0.001)$, pTNM stage $(\mathrm{P}=0.001)$, primary tumor size $(\mathrm{P}=0.008)$, lymph node metastasis $(\mathrm{P}=0.003)$ and tumor location $(\mathrm{P}=0.032)$. No significant relationship was noted between EGFR Del 19 


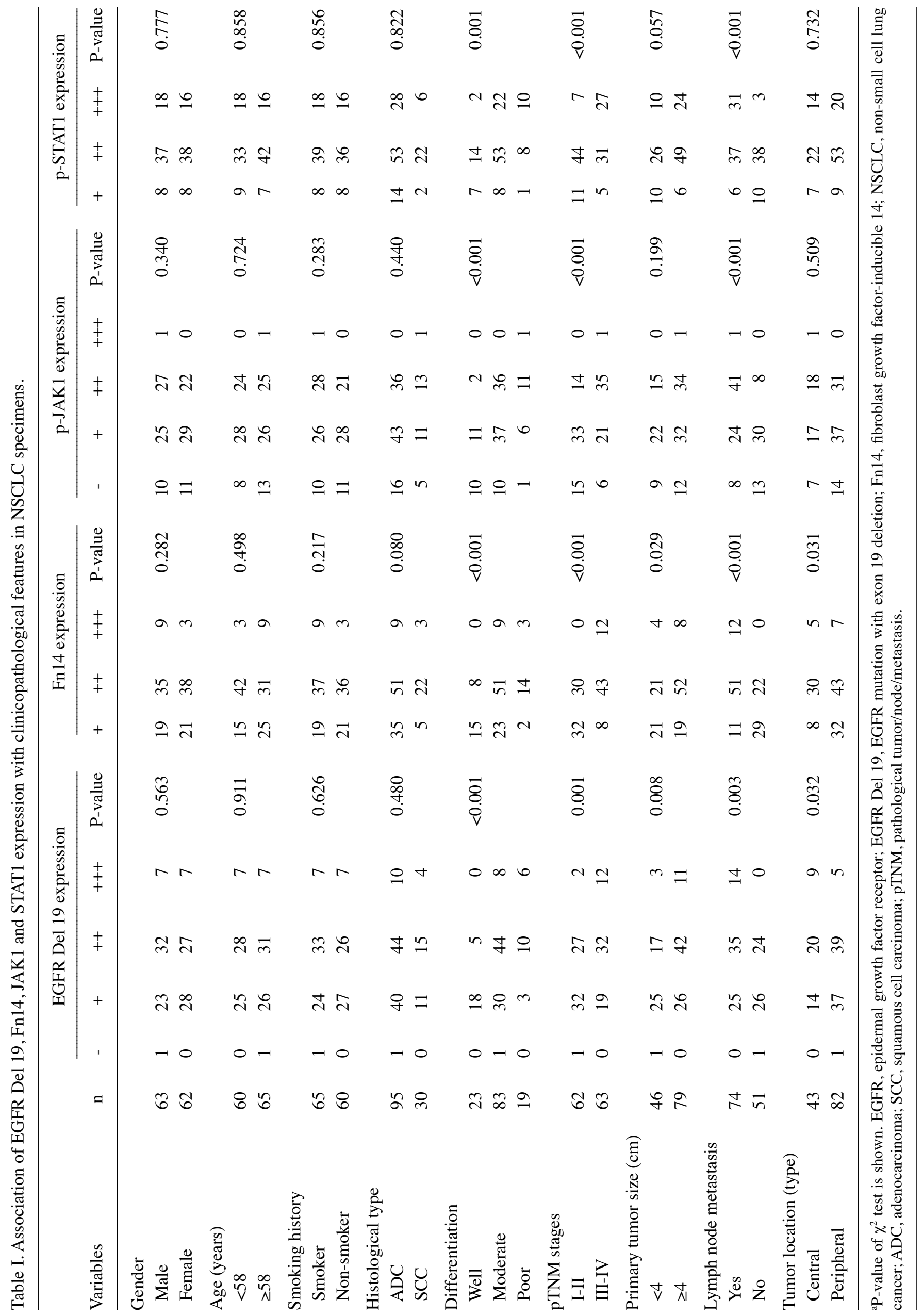


Table II. Fn14 is highly expressed in NSCLC cases with EGFR Del 19.

Fn14 expression

\begin{tabular}{lrrrrrrr}
\cline { 3 - 6 } Group & $\mathrm{n}$ & - & + & ++ & +++ & Positive rate (\%) & P-value \\
\hline EGFR Del 19 samples & 125 & 0 & 43 & 73 & 9 & 100.0 & \\
EGFR wild-type samples & 118 & 61 & 42 & 15 & 0 & 48.3 & \\
EGFR L858R samples & 100 & 28 & 55 & 17 & 0 & 72.0 & $<0.001$ \\
Normal lung samples & 30 & 30 & 0 & 0 & 0 & 0.0 &
\end{tabular}

${ }^{\text {aP-value of }} \chi^{2}$ test is shown. Fn14, fibroblast growth factor-inducible 14; NSCLC, non-small cell lung cancer; EGFR, epidermal growth factor receptor; EGFR Del 19, EGFR mutation with exon 19 deletion.

expression and gender $(\mathrm{P}=0.563)$, age $(\mathrm{P}=0.911)$, smoking history $(\mathrm{P}=0.626)$ and histological type $(\mathrm{P}=0.480)$ (Table I).

Differences in the expression of Fn14 were detected in NSCLC with EGFR Del $19(\mathrm{n}=125)$ and its corresponding normal lung tissues $(n=30)$, EGFR L858R samples $(n=100)$ and EGFR wild-type NSCLC samples $(\mathrm{n}=118)$. A total of $100 \%$ $(125 / 125)$ of tumor sections were classified as Fn14-positive while $100 \%$ (30/30) corresponding normal lung tissue sections were classified as Fn14-negative. Seventy-two percent (72/100) and $48.3 \%$ (57/118) of the tumor sections were classified as Fn14-positive in L858R EGFR and wild-type EGFR NSCLC samples, nevertheless the rate of high expression of Fn14 (++-+++) in Del 19 EGFR, L858R EGFR and wild-type EGFR NSCLC samples was 65.6, 17 and 12.7\%, and there was significant difference in Fn14 expression among EGFR Del 19, L858R EGFR, and wild-type EGFR samples $(\mathrm{P}<0.001)$. Thus, Fn14 was highly expressed in NSCLC with EGFR Del 19 (Table II).

Fn14-positive staining was mainly located on the cell membrane and in the cytoplasm (Fig. 1C and D). In order to evaluate the role of Fn14 in NSCLC, the correlation of Fn14 expression with clinicopathological parameters was investigated (Table I). The results showed that Fn14 expression was significantly associated with differentiation $(\mathrm{P}<0.001)$, pTNM stage $(\mathrm{P}<0.001)$, primary tumor size $(\mathrm{P}=0.029)$, lymph node metastasis $(\mathrm{P}<0.001)$ and tumor location $(\mathrm{P}=0.031)$. No significant relationship was noted between Fn14 expression and gender $(\mathrm{P}=0.282)$, age $(\mathrm{P}=0.498)$, smoking history $(\mathrm{P}=0.217)$ and histological type $(\mathrm{P}=0.080)$.

p-JAK1 $(83.2 \%, 104 / 125)$ (Fig. 1E and F) and p-STAT1 $(100 \%, 125 / 125)$ (Fig. 1G and H) were mainly located in the cytoplasm and cell nucleus. Correlation of JAK1 and STAT1 expression with clinicopathological parameters was then investigated. p-JAK1 and p-STAT1 expression was significantly associated with differentiation $(\mathrm{P}<0.01)$, pTNM stage $(\mathrm{P}<0.001)$ and lymph node metastasis $(\mathrm{P}<0.001)$. No significant relationship was noted with gender, age, smoking history, histological type, primary tumor size and tumor location (Table I).

The mRNA expression levels of Fn14, JAK1 and STAT1 were detected by RT-qPCR. Compared to corresponding normal lung tissues, they were increased in the NSCLC with EGFR Del 19 tissues (Fig. 2). In addition, the protein expression levels of EGFR Del 19, Fn14, p-JAK1 and p-STAT1 were
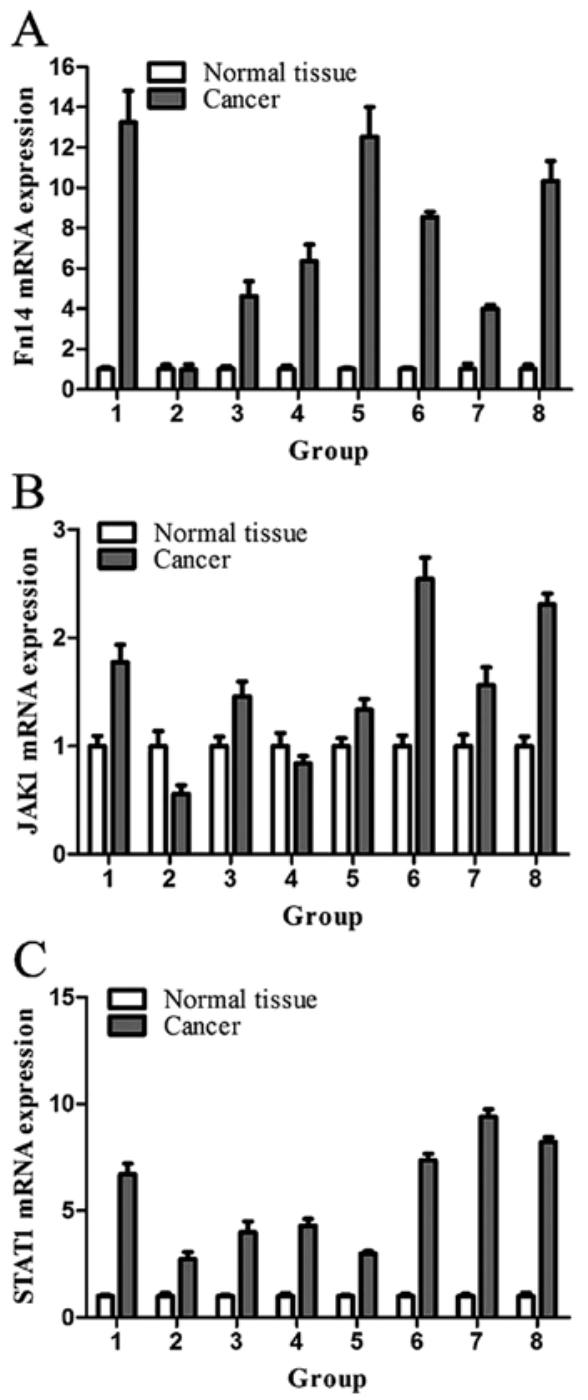

Figure 2. mRNA expression levels of Fn14, JAK1 and STAT1 by RT-qPCR in NSCLC with EGFR Del 19 compared to normal lung tissues. (A) RT-qPCR results of the relative Fn14 expression levels in 8 pairs of NSCLC with EGFR Del 19 and normal lung samples. (B) RT-qPCR results of the relative JAK1 expression levels in 8 pairs of NSCLC with EGFR Del 19 and normal lung samples. (C) RT-qPCR results of the relative STAT1 expression levels in 8 pairs of NSCLC with EGFR Del 19 and normal lung samples.

detected by western blotting in NSCLC with EGFR Del 19 and the corresponding normal lung tissues. The results showed that 


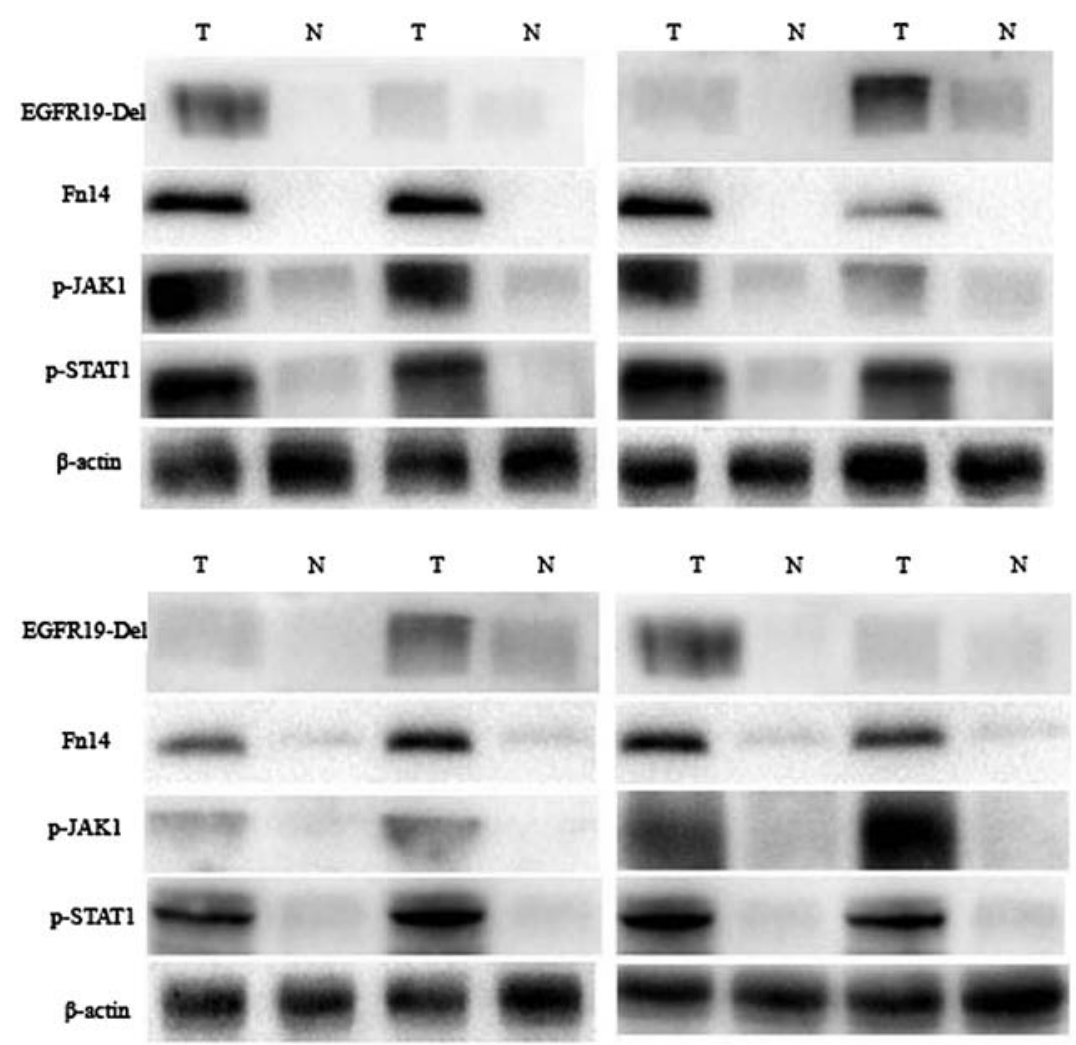

Figure 3. Protein expression levels of EGFR Del 19, Fn14, p-JAK1 and p-STAT1 by western blotting in NSCLC with EGFR Del 19 compared to normal lung tissues. T, tumor; $\mathrm{N}$, normal lung tissue.

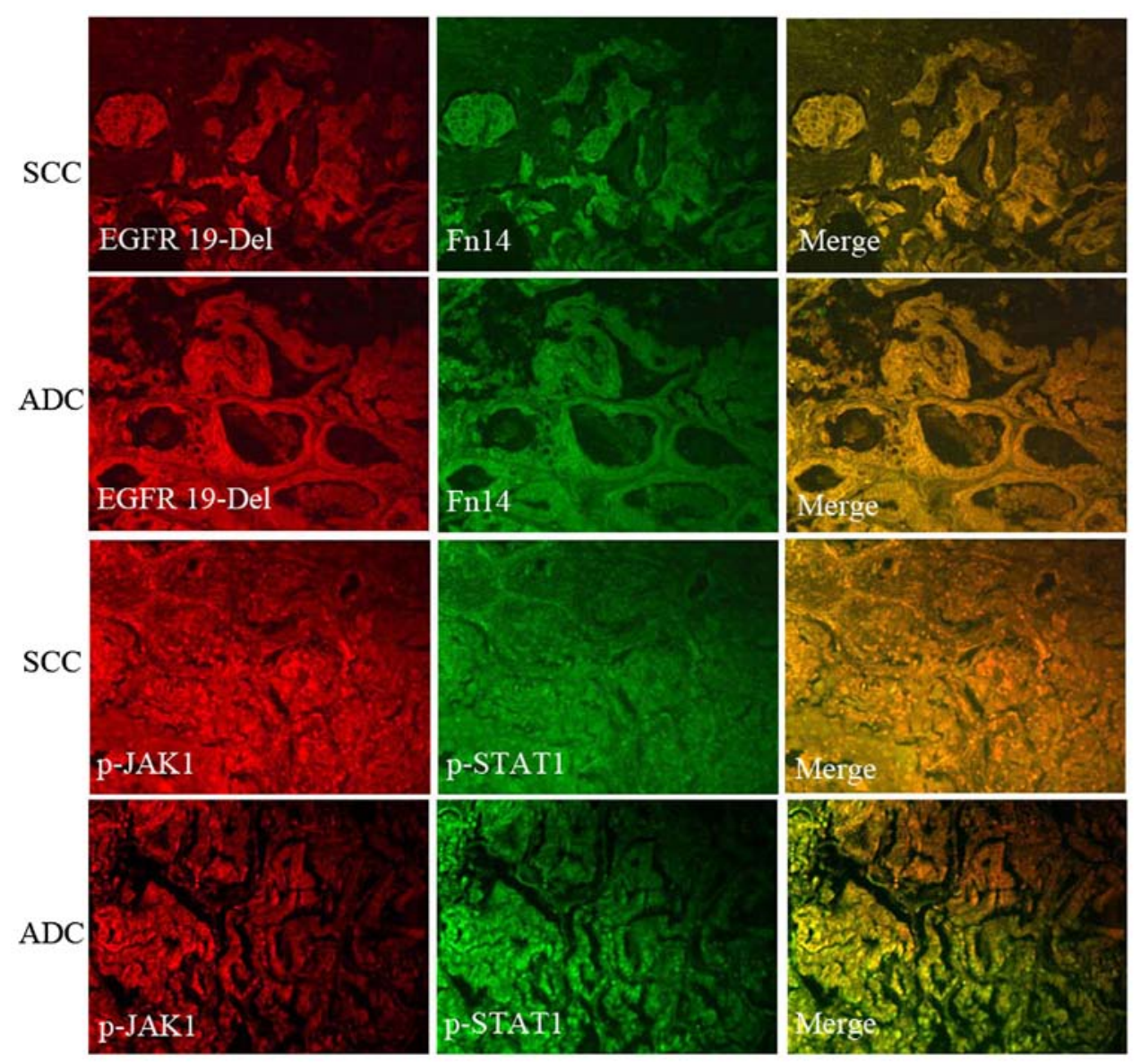

Figure 4. Co-localization of EGFR Del 19, Fn14 protein expression as well as p-JAK1, p-STAT1 protein expression. SCC, squamous cell carcinoma; ADC, adenocarcinoma. 
Table III. The correlation of EGFR Del 19 with Fn14, p-JAK1 and p-STAT1 expression in NSCLC.

\begin{tabular}{|c|c|c|c|c|c|c|c|c|c|c|c|c|c|c|}
\hline \multirow[b]{2}{*}{ Group } & \multirow{2}{*}{$\begin{array}{l}\text { EGFR } \\
\text { Del } 19\end{array}$} & \multicolumn{4}{|c|}{ Fn14 } & \multicolumn{5}{|c|}{ p-JAK1 } & \multicolumn{4}{|c|}{ p-STAT1 } \\
\hline & & + & ++ & +++ & Statistics & - & + & ++ & +++ & Statistics & + & ++ & +++ & Statistics \\
\hline \multicolumn{15}{|c|}{ Histological type } \\
\hline \multirow[t]{4}{*}{ NSCLC } & - & 1 & 0 & 0 & $\chi^{2}=42.814$ & 0 & 1 & 0 & 0 & $\chi^{2}=42.874$ & 1 & 0 & 0 & $\chi^{2}=63.251$ \\
\hline & + & 28 & 20 & 3 & & 16 & 29 & 6 & 0 & $\mathrm{r}_{\mathrm{s}}=0.523$ & 14 & 35 & 2 & $r_{s}=0.620$ \\
\hline & ++ & 11 & 45 & 3 & $r_{s}=0.468$ & 4 & 21 & 34 & 0 & $\mathrm{P}<0.001$ & 1 & 39 & 19 & $\mathrm{P}<0.001$ \\
\hline & +++ & 0 & 8 & 6 & $\mathrm{P}<0.001$ & 0 & 4 & 9 & 1 & & 0 & 1 & 13 & \\
\hline \multirow[t]{4}{*}{$\mathrm{ADC}$} & - & 1 & 0 & 0 & $\chi^{2}=33.152$ & 1 & 0 & 0 & 0 & $\chi^{2}=36.752$ & 1 & 0 & 0 & $\chi^{2}=52.873$ \\
\hline & + & 25 & 13 & 2 & & 12 & 25 & 3 & 0 & $\mathrm{r}_{\mathrm{s}}=0.586$ & 12 & 27 & 1 & $r_{s}=0.665$ \\
\hline & ++ & 9 & 32 & 3 & $r_{s}=0.509$ & 3 & 16 & 25 & 0 & $\mathrm{P}<0.001$ & 1 & 26 & 17 & $\mathrm{P}<0.001$ \\
\hline & +++ & 0 & 6 & 4 & $\mathrm{P}<0.001$ & 0 & 2 & 8 & 0 & & 0 & 0 & 10 & \\
\hline \multirow[t]{3}{*}{ SCC } & + & 3 & 7 & 1 & $\chi^{2}=10.220$ & 4 & 4 & 3 & 0 & $\chi^{2}=12.794$ & 2 & 8 & 1 & $\chi^{2}=12.131$ \\
\hline & ++ & 2 & 13 & 0 & & 1 & 5 & 9 & 0 & $r_{s}=0.377$ & 0 & 13 & 2 & $r_{s}=0.468$ \\
\hline & +++ & 0 & 2 & 2 & $\begin{array}{l}r_{s}=0.314 \\
P=0.037\end{array}$ & 0 & 2 & 1 & 1 & $\mathrm{P}=0.046$ & 0 & 1 & 3 & $\mathrm{P}=0.016$ \\
\hline
\end{tabular}

Differentiation

\begin{tabular}{|c|c|c|c|c|c|c|c|c|c|c|c|c|c|c|}
\hline \multirow[t]{3}{*}{ Well } & + & 12 & 6 & 0 & $\chi^{2}=0.077$ & 8 & 10 & 0 & 0 & $\chi^{2}=8.252$ & 6 & 12 & 0 & $\chi^{2}=7.886$ \\
\hline & ++ & 3 & 2 & 0 & $r_{s}=0.058$ & 2 & 1 & 2 & 0 & $r_{s}=0.212$ & 1 & 2 & 2 & $r_{s}=0.331$ \\
\hline & & & & & $\mathrm{P}=0.782$ & & & & & $\mathrm{P}=0.016$ & & & & $\mathrm{P}=0.019$ \\
\hline \multirow[t]{4}{*}{ Moderate } & - & 1 & 0 & 0 & $\chi^{2}=30.119$ & 1 & 0 & 0 & 0 & $\chi^{2}=24.740$ & 1 & 0 & 0 & $\chi^{2}=39.843$ \\
\hline & + & 15 & 12 & 3 & & 7 & 18 & 5 & 0 & $r_{s}=0.481$ & 7 & 21 & 2 & $r_{s}=0.558$ \\
\hline & ++ & 7 & 35 & 2 & $r_{s}=0.423$ & 2 & 17 & 25 & 0 & $\mathrm{P}<0.001$ & 0 & 31 & 13 & $\mathrm{P}<0.001$ \\
\hline & +++ & 0 & 4 & 4 & $\mathrm{P}<0.001$ & 0 & 2 & 6 & 0 & & 0 & 1 & 7 & \\
\hline \multirow[t]{3}{*}{ Poor } & + & 1 & 2 & 0 & $\chi^{2}=4.087$ & 1 & 1 & 1 & 0 & $\chi^{2}=8.147$ & 1 & 2 & 0 & $\chi^{2}=13.490$ \\
\hline & ++ & 1 & 8 & 1 & $r_{s}=0.436$ & 0 & 3 & 7 & 0 & $r_{s}=0.287$ & 0 & 6 & 4 & $r_{s}=0.729$ \\
\hline & +++ & 0 & 4 & 2 & $\mathrm{P}=0.394$ & 0 & 2 & 3 & 1 & $\mathrm{P}=0.228$ & 0 & 0 & 6 & $\mathrm{P}=0.009$ \\
\hline \multicolumn{15}{|c|}{ TNM stages } \\
\hline \multirow[t]{4}{*}{ I-II } & - & 1 & 0 & 0 & $\chi^{2}=10.446$ & 1 & 0 & 0 & 0 & $\chi^{2}=18.557$ & 1 & 0 & 0 & $\chi^{2}=30.337$ \\
\hline & + & 22 & 10 & 0 & & 10 & 20 & 2 & 0 & $r_{s}=0.440$ & 9 & 23 & 0 & $r_{s}=0.528$ \\
\hline & ++ & 9 & 18 & 0 & $r_{s}=0.406$ & 4 & 13 & 10 & 0 & $\mathrm{P}=0.005$ & 1 & 21 & 5 & $\mathrm{P}<0.001$ \\
\hline & +++ & 0 & 2 & 0 & $\mathrm{P}=0.015$ & 0 & 0 & 2 & 0 & & 0 & 0 & 2 & \\
\hline \multirow[t]{4}{*}{ III-IV } & + & 6 & 10 & 3 & $\chi^{2}=18.102$ & 6 & 9 & 4 & 0 & $\chi^{2}=26.200$ & 5 & 12 & 2 & $\chi^{2}=28.038$ \\
\hline & ++ & 2 & 27 & 3 & & 0 & 8 & 24 & 0 & $\mathrm{r}_{\mathrm{s}}=0.463$ & 0 & 18 & 14 & $r_{s}=0.602$ \\
\hline & +++ & 0 & 6 & 6 & $r_{s}=0.376$ & 0 & 4 & 7 & 1 & $\mathrm{P}=0.001$ & 0 & 1 & 11 & $\mathrm{P}<0.001$ \\
\hline & & & & & $\mathrm{P}<0.001$ & & & & & & & & & \\
\hline
\end{tabular}

${ }^{\text {aP-value of }} \chi^{2}$ test is shown. EGFR, epidermal growth factor receptor; EGFR Del 19, EGFR mutation with exon 19 deletion; Fn14, fibroblast growth factor-inducible 14; NSCLC, non-small cell lung cancer; ADC, adenocarcinoma; SCC, squamous cell carcinoma; pTNM, pathological tumor/node/metastasis.

when compared to the normal lung tissues, Fn14 and p-STAT1 were increased in NSCLC, but this trend was less evident for p-JAK1 protein expression (Fig. 3).

Correlation of EGFR Del 19 with Fn14, p-JAK1, p-STAT1 expression in NSCLC specimens. In order to investigate the correlation of EGFR Del 19 with Fn14, p-JAK1, p-STAT1 expression, the expression rate and intensity of these proteins were analyzed (Table III). The correlation of EGFR Del 19 with Fn14, p-JAK1 and p-STAT1 expression was obvious $(\mathrm{P}<0.05$; $\left.r_{s}>0.3\right)$ in NSCLC, ADC and SCC. The correlation of EGFR Del 19 with Fn14, p-JAK1 and p-STAT1 was high in moderate pathologic stage tissues $\left(\mathrm{P}<0.01 ; \mathrm{r}_{\mathrm{s}}>0.423\right)$. In addition, there were good correlations for TNM stage in NSCLC $(\mathrm{P}<0.05$; $\left.\mathrm{r}_{\mathrm{s}}>0.376\right)$.

Correlation of Fn14 expression with the JAK1/STAT1 pathway in NSCLC specimens with EGFR Del 19. In order to understand the role of Fn14 in NSCLC with EGFR Del 19 and the influence of Fn14 on downstream signaling pathway, correla- 
Table IV. Correlation of Fn14 with p-JAK1 and p-STAT1 expression in NSCLC.

\begin{tabular}{|c|c|c|c|c|c|c|c|c|c|c|}
\hline \multirow[b]{2}{*}{ Group } & \multirow[b]{2}{*}{ Fn14 } & \multicolumn{5}{|c|}{ p-JAK1 } & \multicolumn{4}{|c|}{ p-STAT1 } \\
\hline & & - & + & ++ & +++ & Statistics & + & ++ & +++ & Statistics \\
\hline \multicolumn{11}{|c|}{ Histological type } \\
\hline \multirow[t]{4}{*}{$\mathrm{ADC}$} & + & 12 & 22 & 1 & 0 & $\chi^{2}=33.472$ & 13 & 19 & 3 & $\chi^{2}=30.414$ \\
\hline & ++ & 4 & 19 & 28 & 0 & $\mathrm{r}_{\mathrm{s}}=0.583$ & 1 & 31 & 19 & $\mathrm{r}_{\mathrm{s}}=0.518$ \\
\hline & +++ & 0 & 2 & 7 & 0 & $\mathrm{P}<0.001$ & 0 & 3 & 6 & $\mathrm{P}<0.001$ \\
\hline & + & 2 & 3 & 0 & 0 & $\chi^{2}=16.608$ & 1 & 4 & 0 & $\chi^{2}=12.749$ \\
\hline \multirow[t]{2}{*}{$\mathrm{SCC}$} & ++ & 2 & 8 & 12 & 0 & $r_{s}=0.394$ & 0 & 18 & 4 & $\mathrm{r}_{\mathrm{s}}=0.313$ \\
\hline & +++ & 1 & 0 & 1 & 1 & $\mathrm{P}=0.011$ & 1 & 0 & 2 & $\mathrm{P}=0.013$ \\
\hline \multicolumn{11}{|c|}{ Differentiation } \\
\hline \multirow[t]{3}{*}{ Well } & + & 8 & 6 & 1 & 0 & $\chi^{2}=1.720$ & 7 & 7 & 1 & $\chi^{2}=5.367$ \\
\hline & ++ & 2 & 5 & 1 & 0 & $\mathrm{r}_{\mathrm{s}}=0.268$ & 0 & 7 & 1 & $r_{s}=0.446$ \\
\hline & & & & & & $\mathrm{P}=0.423$ & & & & $\mathrm{P}=0.068$ \\
\hline \multirow[t]{3}{*}{ Moderate } & + & 6 & 17 & 0 & 0 & $\chi^{2}=25.722$ & 6 & 15 & 2 & $\chi^{2}=16.877$ \\
\hline & ++ & 3 & 18 & 30 & 0 & $r_{s}=0.498$ & 1 & 35 & 15 & $\mathrm{r}_{\mathrm{s}}=0.366$ \\
\hline & +++ & 1 & 2 & 6 & 0 & $\mathrm{P}<0.001$ & 1 & 3 & 5 & $\mathrm{P}=0.002$ \\
\hline \multirow[t]{3}{*}{ Poor } & + & 0 & 2 & 0 & 0 & $\chi^{2}=10.939$ & 1 & 1 & 0 & $\chi^{2}=12.350$ \\
\hline & ++ & 1 & 4 & 9 & 0 & $\mathrm{r}_{\mathrm{s}}=0.556$ & 0 & 7 & 7 & $\mathrm{r}_{\mathrm{s}}=0.572$ \\
\hline & +++ & 0 & 0 & 2 & 1 & $\mathrm{P}=0.090$ & 0 & 0 & 3 & $\mathrm{P}=0.015$ \\
\hline \multicolumn{11}{|c|}{ pTNM stages } \\
\hline \multirow[t]{3}{*}{ I-II } & + & 12 & 19 & 1 & 0 & $\chi^{2}=16.396$ & 11 & 19 & 2 & $\chi^{2}=13.053$ \\
\hline & ++ & 3 & 14 & 13 & 0 & $r_{s}=0.494$ & 0 & 25 & 5 & $\mathrm{r}_{\mathrm{s}}=0.424$ \\
\hline & & & & & & $\mathrm{P}<0.001$ & & & & $\mathrm{P}=0.001$ \\
\hline \multirow[t]{3}{*}{ III-IV } & + & 2 & 6 & 0 & 0 & $\chi^{2}=16.980$ & 3 & 4 & 1 & $\chi^{2}=15.647$ \\
\hline & ++ & 3 & 13 & 27 & 0 & $\mathrm{r}_{\mathrm{s}}=0.385$ & 1 & 24 & 18 & $\mathrm{r}_{\mathrm{s}}=0.336$ \\
\hline & +++ & 1 & 2 & 8 & 1 & $\mathrm{P}=0.009$ & 1 & 3 & 8 & $\mathrm{P}=0.004$ \\
\hline
\end{tabular}

${ }^{\text {aP-value of }} \chi^{2}$ test is shown. Fn14, fibroblast growth factor-inducible 14; NSCLC, non-small cell lung cancer; ADC, adenocarcinoma; SCC, squamous cell carcinoma; pTNM, pathological tumor/node/metastasis.

tions between Fn14 expression with JAK1/STAT1 signaling molecules were analyzed (Table IV). There were obvious correlations in ADC and SCC $\left(\mathrm{P}<0.05 ; \mathrm{r}_{\mathrm{s}}>0.3\right)$. Correlations between Fn14 and JAK1 and STAT1 were strong in moderately differentiated tissues $\left(\mathrm{P}<0.01 ; \mathrm{r}_{\mathrm{s}}>0.366\right)$. In addition, there was a good correlation with pTNM stage in NSCLC $\left(\mathrm{P}<0.01 ; \mathrm{r}_{\mathrm{s}}>0.336\right)$.

Co-localization of EGFR Del 19 and Fn14 protein expression as well as p-JAK1 and p-STATl protein expression. EGFR Del 19 and Fn14 were mainly expressed on the cell membrane. In order to further verify whether there is a co-localization phenomenon when they are expressed, immunofluorescence experiments were conducted. Preliminary experimental results showed that the expression of localization phenomenon existed. p-JAK1 and p-STAT1 were mainly located in the cytoplasm and cell nucleus. In addition, the same method was used for co-localization of p-JAK1 and p-STAT1 protein expression. Co-localization phenomenon also existed (Fig. 4).
Correlation of EGFR Del 19 and Fn14 expression with survival in NSCLC with EGFR Del 19 cases. Among factors such as patient age, gender, smoking history, tumor histological type, lymph node metastasis, differentiation and pTNM stages, only the pTNM stage, differentiation and lymph node metastasis were significantly associated with patient survival. The median survival time of patients with pTNM I/II tumors $(n=61)$ was not reached [95\% confidence interval (CI) was not reached], whereas the median survival time of those with TNM III/IV tumors ( $\mathrm{n}=64)$ was 24 months (95\% CI, 17.96-30.04 months; $\mathrm{P}<0.001$; Fig. 5A). Patients with well or moderately differentiated tumors $(n=140$, median survival time, 37.47 months, 95\% CI, 31.73-43.20 months) had a longer survival time than those with poorly differentiated tumors $(n=51$, median survival time, 13 months; 95\% CI, 8.67-17.34 months; $\mathrm{P}<0.001$; Fig. 5B). The median survival time of patients with no lymph node metastasis $(n=74)$ was not reached $(95 \%$ CI was not reached), whereas the median survival time of those with lymph node metastasis $(n=51)$ was 32.07 months $(95 \%$ CI of ratio, 1.3824.733; $\mathrm{P}<0.01$; Fig. 5C). 
A

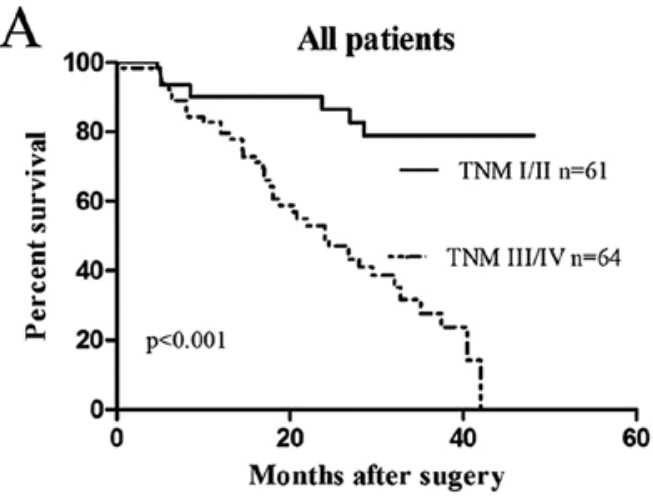

C

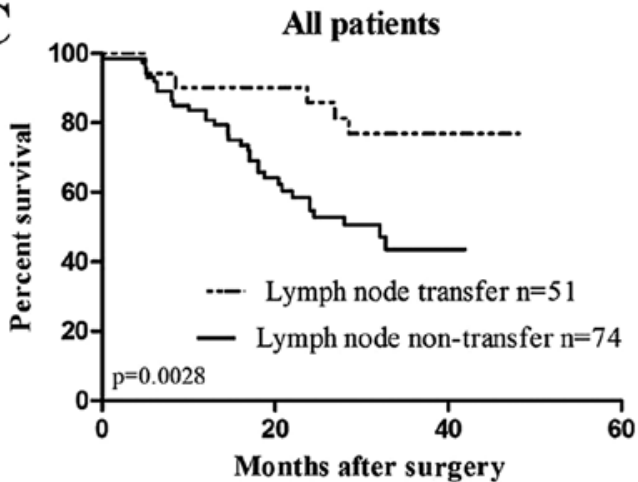

$\mathrm{E}$

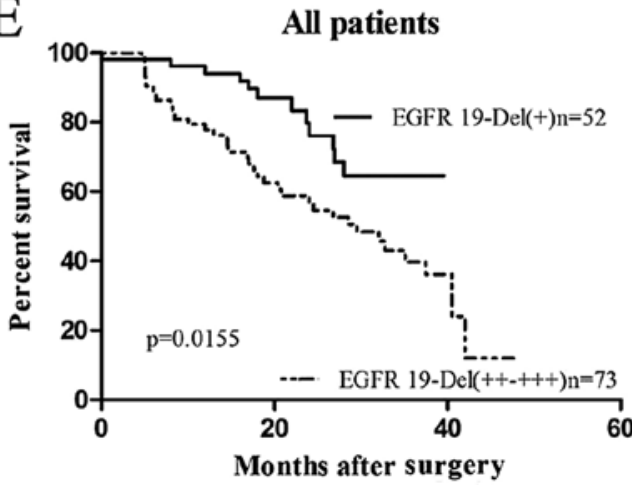

B

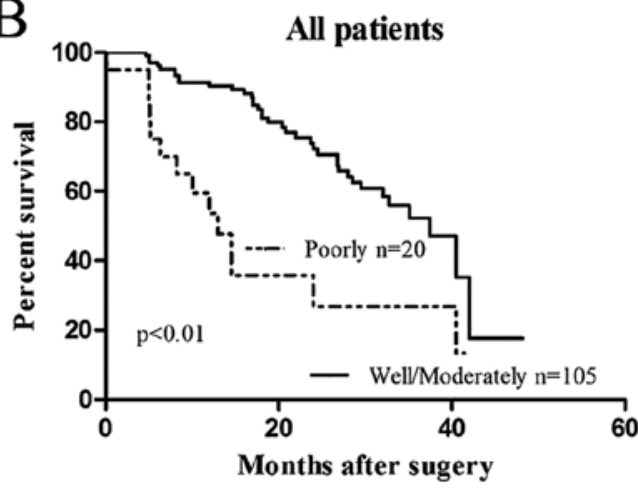

D

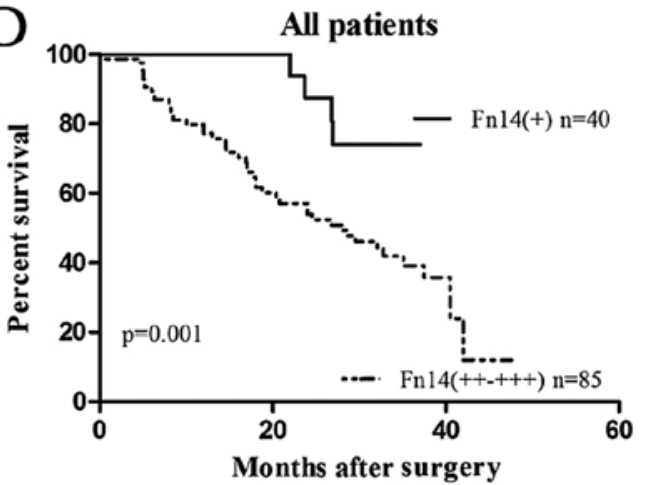

F

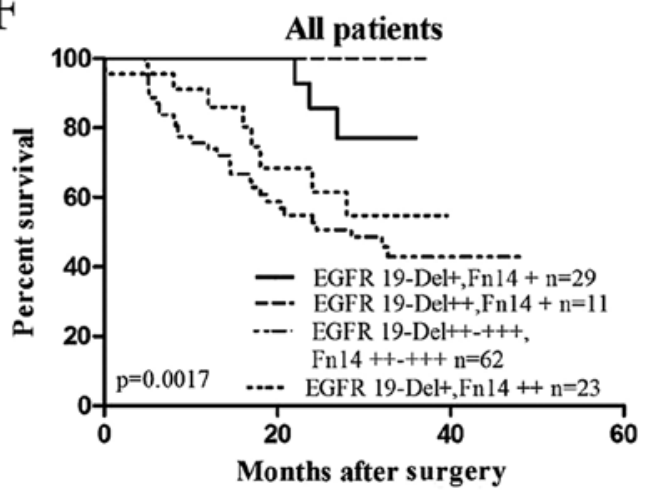

Figure 5. Kaplan-Meier survival analysis of the NSCLC patients. The P-value was determined using the log-rank test. (A) Comparison of the overall survival (OS) between pTNM I/II and pTNM III/IV NSCLC patients. (B) Comparison of the OS of patients between well/moderately differentiated and poorly differentiated NSCLC tissues. (C) Comparison of OS between lymph node non-metastatic and lymph node metastatic NSCLC patients. (D) Comparison of OS between Fn14 weakly positive and Fn14 highly positive NSCLC patients. (E) Comparison of the OS between EGFR Del 19 weakly positive and EGFR Del 19 highly positive NSCLC patients. (F) Comparison of the OS between EGFR Del 19, Fn14 weakly positive and EGFR Del 19, Fn14 highly positive NSCLC patients.

To investigate the relationship between EGFR Del 19 and Fn14 expression and the clinical outcome of NSCLC patients, the correlation of EGFR Del 19 and Fn14 expression with patient survival and EGFR Del 19, Fn14 expression status were analyzed. Patients with Fn14 high positive expression had a significantly worse prognosis than those with Fn14 low positive expression $(\mathrm{P}=0.001$; Fig. $5 \mathrm{D})$. The median survival time of patients with Fn14 high positive NSCLC $(n=85)$ was 28 months (95\% CI, 17.99-38.01 months) and the mean survival time was 27.07 months (95\% CI, 23.49-30.66 months), whereas the median survival time of patients with Fn14 low positive NSCLC $(n=40)$ was not reached and the mean survival time was 33.86 months (95\% CI, 31.17-36.55 months). Patients with high positive expression of EGFR Del 19 had a significantly worse prognosis than those with low positive expression of EGFR Del 19 ( $\mathrm{P}=0.0155$; Fig. 5E). The median survival time of patients with EGFR Del 19 low positive NSCLC ( $\mathrm{n}=52)$ was 32.8 months (95\% CI of ratio, 0.2551-0.8663), whereas the median survival time of patients with EGFR Del 19 high positive NSCLC ( $\mathrm{n}=73$ ) was not reached. Comprehensive statistics were analyzed concerning the influence of EGFR Del 19 and Fn14 on patient prognosis. We found that with increased expression of EGFR Del 19 and Fn14, the shorter the patient survival ( $\mathrm{P}<0.01$; Fig. 5F).

To further assess whether EGFR Del 19 and Fn14 expression represents a common prognostic parameter in NSCLC patients with EGFR Del 19, regression analysis using the Cox's proportional hazards model was performed. The covariate parameters included several clinicopathological factors in addition to EGFR Del 19 and Fn14, as shown in Table V. In the univariate analysis, factors including EGFR Del 19 expression, Fn14 expression, pTNM stage and differ- 
Table V. Cox proportional hazards model analysis of variables affecting survival in NSCLC patients.

\begin{tabular}{|c|c|c|c|c|c|}
\hline \multirow[b]{2}{*}{ Variables } & \multirow[b]{2}{*}{ Categories } & \multicolumn{2}{|c|}{ Univariate analysis } & \multicolumn{2}{|c|}{ Multivariate analysis } \\
\hline & & $\mathrm{HR}(95 \% \mathrm{CI})$ & $\mathrm{P}$-value & $\mathrm{HR}(95 \% \mathrm{CI})$ & P-value \\
\hline Age (years) & $\geq 58 /<58$ & $0.751(0.428-1.318)$ & 0.319 & & \\
\hline Gender & Male/female & $1.031(0.592-1.796)$ & 0.914 & & \\
\hline Smoking history & Smoking/non-smoking & $1.001(0.573-1.747)$ & 0.998 & & \\
\hline Histological type & SCC/ADC & $0.712(0.383-1.323)$ & 0.282 & & \\
\hline Differentiation & Poor/well+moderate & $0.291(0.154-0.549)$ & $<0.001$ & $0.424(0.218-0.824)$ & 0.011 \\
\hline pTNM stage & I/II/III/IV & $0.222(0.108-0.457)$ & $<0.001$ & $0.350(0.160-0.768)$ & 0.009 \\
\hline Fn14 expression & High positive/low positive & $0.183(0.066-0.512)$ & 0.001 & $0.316(0.102-0.983)$ & 0.047 \\
\hline EGFR Del 19 & High positive/low positive & $0.430(0.223-0.829)$ & 0.012 & $1.065(0.508-2.233)$ & 0.867 \\
\hline
\end{tabular}

NSCLC, non-small cell lung cancer; HR, hazard ratio; CI, confidence interval; SCC, squamous cell carcinoma; ADC, adenocarcinoma; pTNM, pathological tumor/node/metastasis; Fn14, fibroblast growth factor-inducible 14; EGFR, epidermal growth factor receptor; EGFR Del 19, EGFR mutation with exon 19 deletion.

entiation showed a significantly higher hazard ratio for poor prognosis.

Moreover, multivariate analysis was carried out using the significant factors observed in the univariate analysis. The results showed that, in addition to TNM stage and differentiation, Fn14 expression was an independent prognostic factor $(\mathrm{P}=0.047$; Table V). These results strongly indicate that Fn14 expression in NSCLC patients is closely related to poor prognosis.

\section{Discussion}

Normally, most NSCLC patients carry EGFR mutations, and EGFR Del 19 is one of the most common forms. Although NSCLC patients with EGFR Del 19 are sensitive to EGFR TKI, acquired resistance is inevitable to limiting the therapeutic effects in patients. Researchers have conjectured whether NSCLC carcinogenesis is related with EGFR Del 19 and whether the EGFR Del 19 mutation may upregulate new downstream signaling pathways or promote a novel molecular expression, and this issue is unclear. Thus, we aimed to ascertain whether the pathogenesis of NSCLC is associated with EGFR Del 19.

Increasing evidence shows that Fn14 is weakly expressed in healthy tissues (7-9), but is highly expressed in contexts of tissue damage caused by hypoxia, oxidative stress and tumor growth (10-14). Thus, researchers have increasingly given more attention to the function of Fn14 in cancers and found that Fn14 expression may be a valuable potential biomarker (20-22). In NSCLC cell lines, Fn14 expression levels may be correlated with EGFR mutations (16), and it may function in cancer through the JAK/STAT1 pathway. Although STAT1 is well known as a master transcription factor for IFN-related intracellular signaling, leading to antiviral activity (23-26), emerging data has revealed that in certain cellular contexts the IFN/STAT1 pathway may facilitate tumor cell growth $(27,28)$. There is limited research concerning the functions among EGFR Del 19, Fn14 and the JAK/STAT pathway. Thus, in the present study, we assessed the correlation of EGFR Del 19 with Fn14 and the JAK/STAT pathway.
In the present study, we performed IHC, RT-qPCR and western blot analysis on surgically resected NSCLC specimens to investigate EGFR Del 19, Fn14, JAK1 and STAT1 expression. IHC and western blotting results showed that compared to EGFR L858R, EGFR wild-type NSCLC tissues and corresponding normal lung tissues, Fn14 protein expression was significantly higher in NSCLC with EGFR Del 19. Fn14, JAK1 and STAT1 were increased at the mRNA levels and were associated with clinicopathological characteristics, such as pTNM stage, differentiation, lymph node metastasis, but not with age, gender, smoking history and histological subtypes.

The present study was the first to investigate the correlation of EGFR Del 19 with Fn14 and JAK1/STAT1 pathway. The correlations of EGFR Del 19 with Fn14, p-JAK1, p-STAT1 and Fn14 with p-JAK1, p-STAT1 were significant in regards to histological type, differentiation and pTNM stage $\left(r_{s}>0.3\right.$; $\mathrm{P}<0.05)$. From the data, we found that both EGFR Del 19 and Fn14 were associated with p-JAK1 and p-STAT1. This indicates that there is better relationship among these four factors. In addition, EGFR mutations in NSCLC can selectively activate signaling pathways which promote cell survival and induce proliferation such as STAT (29). Thus, this is a substantial proof which can testify the relationship between EGFR Del 19 and p-JAK1 and p-STAT1. Furthermore, one study showed that Fn14 expression was higher in EGFR Del 19 cell lines, and erlotinib (one of TKIs) inhibited Fn14 expression (16). Thus, we may infer that EGFR Del 19 may upregulate Fn14, JAK1 and STAT1 protein expression, but the detailed mechanism of action is not clear. In order to further verify whether there is a co-localization phenomenon when EGFR Del 19 and Fn14 are expressed, immunofluorescence experiments were conducted. Preliminary experimental results showed that a co-localization phenomenon existed. All these signs indicated that EGFR Del 19 may play an important role in migration, invasion and metastasis of NSCLC and EGFR Del 19 may upregulate Fn14 and JAK1/STAT1 pathway to promote NSCLC progression.

In addition, to the best of our knowledge, the present study was the first to indicate an association between EGFR Del 19 
and Fn14 positivity in NSCLC patients and poor prognosis, and these factors are pivotal to affecting patient prognosis. The results of survival analysis suggested that Fn14 may be an independent prognostic factor of NSCLC patients. Due to the limitations of the sample size and follow-up, the median survival time in many groups could not be obtained. To compensate for these shortcomings, we intend to carry out further multicenter clinical studies, expand the sample size and enrich the means of detection.

Taken together with the results of the present study, we believe that EGFR Del 19 may promote NSCLC progression through upregulation of Fn14 and the JAK1/STAT1 pathway, and Fn14 may be a potential biomarker for NSCLC with EGFR Del 19 patients. However, the mechanism of NSCLC progression by EGFR Del 19 upregulating Fn14 and JAK/ STAT pathway needs to be further examined.

In conclusion, we showed that a high proportion of NSCLC with EGFR Del 19 expressed Fn14, JAK1 and STAT1 and the expression of these proteins was significantly associated with differentiation, pTNM stage and lymphatic metastasis. Moreover, levels of Fn14, JAK1 and STAT1 expression were related with EGFR Del 19, and there was a co-localization phenomenon when EGFR Del 19 and Fn14 were expressed. Thus, our results indicate that EGFR Del 19 may promote Fn14 and JAK1/STAT1 expression in NSCLC, and Fn14 has a significant role in NSCLC with EGFR Del 19, and may serve as a prognostic biomarker in NSCLC with EGFR Del 19.

\section{Acknowledgements}

We thank Liangbo Fan, Peng Chen and Guang Yang (Thoracic Surgery Department Laboratory) for their help in providing experimental technical support. Finally, we would like to thank all members of our research team, who collaborated to ensure that the study proceeded smoothly.

\section{References}

1. Devesa SS, Bray F, Vizcaino AP and Parkin DM: International lung cancer trends by histologic type: Male:female differences diminishing and adenocarcinoma rates rising. Int J Cancer 117: 294-299, 2005 .

2. Nicholson RI, Gee JM and Harper ME: EGFR and cancer prognosis. Eur J Cancer 37 (Suppl 4): S9-S15, 2001.

3. Krause DS and Van Etten RA: Tyrosine kinases as targets for cancer therapy. N Engl J Med 353: 172-187, 2005.

4. Hynes NE and Lane HA: ERBB receptors and cancer: The complexity of targeted inhibitors. Nat Rev Cancer 5: 341-354, 2005.

5. Paez JG, Jänne PA, Lee JC, Tracy S, Greulich H, Gabriel S, Herman P, Kaye FJ, Lindeman N, Boggon TJ, et al: EGFR mutations in lung cancer: Correlation with clinical response to gefitinib therapy. Science 304: 1497-1500, 2004.

6. Kaneda T, Hata A, Tomioka H, Tanaka K, Kaji R, Fujita S, Tomii K and Katakami N: Possible differential EGFR-TKI efficacy among exon 19 deletional locations in EGFR-mutant non-small cell lung cancer. Lung Cancer 86: 213-218, 2014.

7. Girgenrath $M$, Weng S, Kostek CA, Browning B, Wang M, Brown SA, Winkles JA, Michaelson JS, Allaire N, Schneider P, et al: TWEAK, via its receptor Fn14, is a novel regulator of mesenchymal progenitor cells and skeletal muscle regeneration. EMBO J 25: 5826-5839, 2006

8. Baxter FO, Came PJ, Abell K, Kedjouar B, Huth M, Rajewsky K, Pasparakis $M$ and Watson CJ: IKKbeta/2 induces TWEAK and apoptosis in mammary epithelial cells. Development 133: 3485-3494, 2006

9. Winkles JA: The TWEAK-Fn14 cytokine-receptor axis: Discovery, biology and therapeutic targeting. Nat Rev Drug Discov 7: 411-425, 2008.
10. Burkly LC, Michaelson JS and Zheng TS: TWEAK/Fn14 pathway: An immunological switch for shaping tissue responses. Immunol Rev 244: 99-114, 2011.

11. Kim SH, Kang YJ, Kim WJ, Woo DK, Lee Y, Kim DI, Park YB, Kwon BS, Park JE and Lee WH: TWEAK can induce pro-inflammatory cytokines and matrix metalloproteinase-9 in macrophages. Circ J 68: 396-399, 2004

12. Pettersen I, Baryawno N, Abel F, Bakkelund WH, Zykova SN Winberg JO, Moens U, Rasmuson A, Kogner P, Johnsen JI, et al: Expression of TWEAK/Fn14 in neuroblastoma: implications in tumorigenesis. Int J Oncol 42: 1239-1248, 2013.

13. Lynch CN, Wang YC, Lund JK, Chen YW, Leal JA and Wiley SR: TWEAK induces angiogenesis and proliferation of endothelial cells. J Biol Chem 274: 8455-8459, 1999.

14. Harada N, Nakayama M, Nakano H, Fukuchi Y, Yagita H and Okumura K: Pro-inflammatory effect of TWEAK/Fn14 interaction on human umbilical vein endothelial cells. Biochem Biophys Res Commun 299: 488-493, 2002.

15. Chicheportiche Y, Chicheportiche R, Sizing I, Thompson J, Benjamin CB, Ambrose C and Dayer JM: Proinflammatory activity of TWEAK on human dermal fibroblasts and synoviocytes: Blocking and enhancing effects of anti-TWEAK monoclonal antibodies. Arthritis Res 4: 126-133, 2002.

16. Whitsett TG, Cheng E, Inge L, Asrani K, Jameson NM, Hostetter G, Weiss GJ, Kingsley CB, Loftus JC, Bremner R, et al: Elevated expression of Fn14 in non-small cell lung cancer correlates with activated EGFR and promotes tumor cell migration and invasion. Am J Pathol 181: 111-120, 2012.

17. Chapman MS, Wu L, Amatucci A, Ho SN and Michaelson JS: TWEAK signals through JAK-STAT to induce tumor cell apoptosis. Cytokine 61: 210-217, 2013.

18. Gastl G, Spizzo G, Obrist P, Dünser M and Mikuz G: Ep-CAM overexpression in breast cancer as a predictor of survival. Lancet 356: 1981-1982, 2000.

19. Zhao J, Zhou Y, Zhang Z, Tian F, Ma N, Liu T, Gu Z and Wang Y: Upregulated fascin1 in non-small cell lung cancer promotes the migration and invasiveness, but not proliferation. Cancer Lett 290: 238-247, 2010.

20. Kwon OH, Park SJ, Kang TW, Kim M, Kim JH, Noh SM, Song KS, Yoo HS, Wang Y, Pocalyko D, et al: Elevated fibroblast growth factor-inducible 14 expression promotes gastric cancer growth via nuclear factor- $\kappa \mathrm{B}$ and is associated with poor patient outcome. Cancer Lett 314: 73-81, 2012.

21. Zhou H, Ekmekcioglu S, Marks JW, Mohamedali KA, Asrani K, Phillips KK, Brown SA, Cheng E, Weiss MB, Hittelman WN, et al: The TWEAK receptor Fn14 is a therapeutic target in melanoma: Immunotoxins targeting Fn14 receptor for malignant melanoma treatment. J Invest Dermatol 133: 1052-1062, 2013.

22. Huang M, Narita S, Tsuchiya N, Ma Z, Numakura K, Obara T, Tsuruta H, Saito M, Inoue T, Horikawa Y, et al: Overexpression of Fn14 promotes androgen-independent prostate cancer progression through MMP-9 and correlates with poor treatment outcome. Carcinogenesis 32: 1589-1596, 2011.

23. Townsend PA, Scarabelli TM, Davidson SM, Knight RA, Latchman DS and Stephanou A: STAT-1 interacts with p53 to enhance DNA damage-induced apoptosis. J Biol Chem 279: 5811-5820, 2004.

24. Stephanou A, Brar BK, Knight RA and Latchman DS: Opposing actions of STAT- 1 and STAT- 3 on the Bcl- 2 and Bcl-x promoters. Cell Death Differ 7: 329-330, 2000.

25. Chin YE, Kitagawa M, Kuida K, Flavell RA and Fu XY: Activation of the STAT signaling pathway can cause expression of caspase 1 and apoptosis. Mol Cell Biol 17: 5328-5337, 1997.

26. Kumar A,Commane M,Flickinger TW, Horvath CM and Stark GR: Defective TNF-alpha-induced apoptosis in STAT1-null cells due to low constitutive levels of caspases. Science 278: 1630-1632, 1997.

27. Fryknäs M, Dhar S, Oberg F, Rickardson L, Rydåker M, Göransson H, Gustafsson M, Pettersson U, Nygren P, Larsson R, et al: STAT1 signaling is associated with acquired crossresistance to doxorubicin and radiation in myeloma cell lines. Int J Cancer 120: 189-195, 2007.

28. Roberts D, Schick J, Conway S, Biade S, Laub PB, Stevenson JP, Hamilton TC, O'Dwyer PJ and Johnson SW: Identification of genes associated with platinum drug sensitivity and resistance in human ovarian cancer cells. Br J Cancer 92: 1149-1158, 2005.

29. Zimmer S, Kahl P, Buhl TM, Steiner S, Wardelmann E, Merkelbach-Bruse S, Buettner R and Heukamp LC: Epidermal growth factor receptor mutations in non-small cell lung cancer influence downstream Akt, MAPK and Stat3 signaling. J Cancer Res Clin Oncol 135: 723-730, 2009. 